\title{
Inverse hydrogen bond: theoretical investigation on the nature of interaction and spectroscopic properties
}

\author{
Agnieszka Ilnicka $\cdot$ Joanna Sadlej
}

Received: 24 June 2011 / Accepted: 19 August 2011/Published online: 9 June 2012

(C) The Author(s) 2012. This article is published with open access at Springerlink.com

\begin{abstract}
A computational investigation was carried out to characterize the inverse (hydride) hydrogen bond in model complexes. Properties such as molecular structures and energetics have been studied by supermolecular MP2 approach. We focus on vibrational spectra, NMR shielding, and spin-spin coupling constants-signals that reflect the electronic structures of the compounds. The bonding in these complexes has been analyzed for a first time by SymmetryAdapted Perturbation Theory to provide the intricate insight into the nature of the interaction. The cyclic complexes, which have multiple interaction, are stable due to strong redistribution of electron density upon the complexation and differ from the linear ones by the induction energy as the most important term exceeding the electrostatic term. The linear complexes, which represent the inverse hydrogen bond, are characterized by much stronger induction than dispersion energy, contrary to the conventional hydrogen bonds, where these two terms happen to be of nearly equal magnitude. This result is the most noticeable difference between inverse and conventional hydrogen bonds.
\end{abstract}

Keywords Inverse hydrogen bond - SAPT .

AIM analysis · NMR parameters

\section{Introduction}

There is no doubt in how essential role the hydrogen bonds have in many areas of chemistry and biochemistry. They are

This paper is dedicated to Professor Malgorzata Witko.

A. Ilnicka · J. Sadlej $(\bowtie)$

Department of Chemistry, University of Warsaw, Pasteura 1,

02-093 Warsaw, Poland

e-mail: sadlej@chem.uw.edu.pl responsible for the structure of proteins, the stability of DNA but also have their significant role in forming the crystallographic structures. Despite the fact that it is obvious that hydrogen bond is important and interesting topic of research, there are still problems in defining what exactly the hydrogen bond is. Hydrogen bonds cover a broad range, from very strong to weak, having energies slightly above van der Waals interactions. Its energy cannot be measured directly; it can be only estimated. The IUPAC recommends is using such name for the form of association $\mathrm{X}-\mathrm{H} \cdots \mathrm{Y}$ between the electronegative atom $\mathrm{Y}$ and the hydrogen atom attached to the second atom $\mathrm{X}$, and in which the electrostatic interactions play the key role [1]. Such definition does not cover all interactions in which the hydrogen atom is an essential element, but new, less restricted definition was published recently [2].

In spite of the classical HBs are still mostly investigated, papers with theoretical and experimental study on this unconventional ones have occurred as well. The unconventional hydrogen bonds can be classified to four groups:

1. HBs with unconventional $\mathrm{H}$ donors, such as $\mathrm{C}-\mathrm{H}$,

2. HBs with unconventional $\mathrm{H}$ acceptors, such as $\pi$-bonded groups,

3. dihydrogen bonds $\mathrm{Y}-\mathrm{H} \cdots \mathrm{H}-\mathrm{X}$,

4. inverse (or hydride) hydrogen bonds.

In significant majority of $\mathrm{HBs}$, the $\mathrm{X}-\mathrm{H}$ moiety is the hydrogen-bond donor (i.e., electron acceptor), while $\mathrm{Y}$ moiety play a hydrogen-bond acceptor role (i.e., electron donor), according to the scheme $\mathrm{X}^{-}-\mathrm{H} \cdots \mathrm{Y}^{+}$. The situation changes in the dihydrogen bonds (DHB), where one of the hydrogen atoms provides and the second accepts the electrons, forming $\mathrm{X}^{+}-\mathrm{H} \cdots \mathrm{H}-\mathrm{Y}^{-}[3,4]$. The third situation occurs in the case of so-called inverse hydrogenbonded (InHB) complexes $\mathrm{X}^{+} \mathrm{H} \cdots \mathrm{Y}^{-}$, where the hydrogen atom rich in electron density and situated among 

$\mathrm{F} 2 \frac{1.420}{\mathrm{Be}} \frac{1.420}{\mathrm{~F} 1}$
$\mathrm{H} 2 \stackrel{1.336}{\mathrm{Be}} \frac{1.336}{\mathrm{H} 1}$
$\mathrm{Li} \stackrel{1.610}{\mathrm{~F}}$
H $\stackrel{1.619}{\mathrm{Li}}$

L1 H2 $\frac{1.326}{B e} \frac{1.338}{H}$ H - $1.879--$ Li $\frac{1.622}{F}$
L2 H3 $\stackrel{1.623}{\longrightarrow}$ Li -. 1.882 -- H1 $\frac{1.337}{\mathrm{Be}} \stackrel{1.326}{\mathrm{H} 2}$
C1

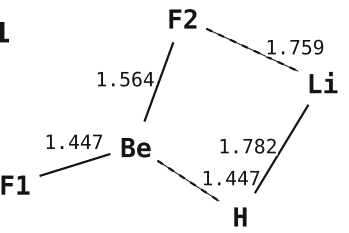

$A(F 1, B e, F 2)=127.329$

$A(F 1, B e, H)=129.491$

$A(F 2, B e, H)=103.180$

$\mathrm{A}(\mathrm{F} 2, \mathrm{Li}, \mathrm{H})=83.585$
C2

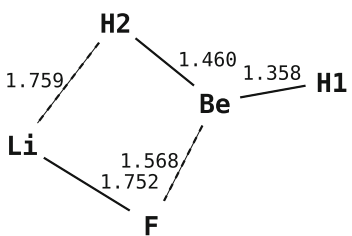

$\mathrm{A}(\mathrm{H} 1, \mathrm{Be}, \mathrm{H} 2)=130.852$

$\mathrm{A}(\mathrm{H1}, \mathrm{Be}, \mathrm{F})=127.217$

$A(H 2, B e, F)=101.93$

$A(H 2, L i, F)=84.155$

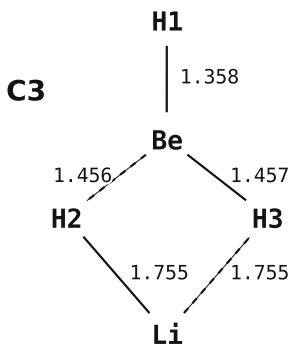

$A(H 1, B e, L i)=179.949$

$A(B e, L i, H 2)=40.881$

$A(B e, L i, H 3)=40.890$

$A(H 2, L i, H 3)=81.771$

Fig. 1 The geometry of monomers and complexes (calculated using MP2/aug-cc-pVDZ). The numbers in $\AA$, A in degree

electropositive centers can provide a formation of HB with non-hydrogen, usually the alkaline atoms [5]. In general, conventional hydrogen-bond $\mathrm{X}-\mathrm{H}$ moiety is define by $\mathrm{X}$, which is more electronegative than $\mathrm{H}$ (i.e., $\mathrm{O}-\mathrm{H} \cdots \mathrm{B}$, $\mathrm{N}-\mathrm{H} \cdots \mathrm{B}$ ), while proton acceptor part B posses, for instant, electron pairs, as $\mathrm{N}, \mathrm{O}$, or $\mathrm{F}$ atoms. To study InHB nonconventional hydrogen bond we chose the molecule $\mathrm{BeH}_{2}$ as an electron donor (with heavy electron-deficient atom), while $\mathrm{LiH}$ or $\mathrm{LiF}$ played a role of electron acceptor molecules [5]. The transfer of charge is thus in the same direction as the proton flow (i.e., from $\mathrm{X}-\mathrm{H}$ to $\mathrm{Y}$ ); therefore this interaction was called "inverse," contrary to the conventional hydrogen bond (where transfer of charges is from the moiety $\mathrm{Y}$ to $\mathrm{X}-\mathrm{H}$ moiety) [5, 6]. Such bonds normally have energy of -5 to $-10 \mathrm{kcal} \mathrm{mol}^{-1}$. In contrary to a huge number of papers subjected on the conventional hydrogen bonds, there are hardly few papers on the inverse ones, and they provide mainly information based on the Bader analysis of electron density, the Atoms in Molecules approach (AIM) [5, 6].

This paper is, to the best of authors' knowledge, the first presenting the analysis of the inverse hydrogen bonds given by the Symmetry-Adapted Perturbation Theory (SAPT), which is the powerful tool to look into the nature of the intermolecular interaction [7]. The aim of the paper is also to perform the computations of spectroscopic properties (vibrational and NMR parameters), as molecular spectroscopy represents an important (and sometimes the only) method for the detection and characterization of hydrogen bonds and other intermolecular interactions. Specially, the NMR spectra are sensitive probes of the electronic structure of molecules. These parameters are also sensitive to intermolecular interactions. The most widely used parameter in those studies is the isotropic shielding of the proton involved in the hydrogen bond.

\section{Computational details}

Geometry optimization and supermolecular energy calculations

The molecules and complexes on which the investigation was proceeded are shown in the Fig. 1. There are two linear complexes with the inverse hydrogen bond and three cyclic complexes-one with two hydrogen atoms involved in forming complex, and two with the hydrogen and fluorine atoms involved. These model complexes and their monomers were optimized on the second order of Möller-Plesset perturbation theory (MP2) with the aug-cc-pVDZ basis set. All stationary points the vibration frequencies were calculated to confirm the minimum-in all cases there were all positive frequencies. The aug-cc-pVDZ basis set is now accepted for studying geometry of molecules and interacting systems on the correlated level. The supermolecular interaction energy was obtained by substracting the energies of the monomers from the energy of the complex. The computed interaction energies were corrected for a basis set superposition error (BSSE) following the prescription of Boys and Bernardi [8]. They are presented in the tables under the name of the binding energy, $D_{\mathrm{e}}$. To relate the calculated interaction energy to the observed dissociation 
energy $D_{0}$, a correction for the zero-point vibrational (ZPV) energy of the complex and the monomers was added. The ZPV correction was calculated in the harmonic approximation at the respective level of theory. The geometry optimization as well as the calculations of vibrational frequencies and interaction energies were carried out using the Gaussian 03 program [9].

\section{Symmetry-Adapted Perturbation Theory}

The SAPT approach is the powerful tool to investigate the intermolecular interaction. Is now a mature and routinely used method. In contrary to the supermolecular calculations, it provides not only the total interaction energy, but also gives insight into the nature of interaction and provide a clear physical picture of the interactions. In this study, second order SAPT (SAPT2) and full SAPT approximations were employed $[10,11]$.

The interaction energy at the SAPT2 approach is defined by the main four terms: electrostatic, exchange, induction, dispersion and additional, last component:

$E_{\text {int }}^{\mathrm{SAPT} 2}=E_{\mathrm{elst}}+E_{\mathrm{exch}}+E_{\mathrm{ind}}+E_{\mathrm{disp}}+\delta E_{\mathrm{int}, \mathrm{resp}}^{\mathrm{HF}}$

where

$$
\begin{aligned}
E_{\mathrm{elst}} & =E_{\text {elst }}^{(10)}+E_{\text {elst,resp }}^{(12)} \\
E_{\text {exch }} & =E_{\text {exch }}^{(10)}+E_{\text {exch }}^{(11)}+E_{\text {exch }}^{(12)} \\
E_{\text {ind }} & =E_{\text {ind,resp }}^{(20)}+E_{\text {exch-ind,resp }}^{(20)}+{ }^{t} E_{\text {ind }}^{(22)}+{ }^{t} E_{\text {exch-ind }}^{(22)} \\
E_{\text {disp }} & =E_{\text {disp }}^{(20)}+E_{\text {exch-disp }}^{(20)} \\
\delta E_{\text {int,resp }}^{\mathrm{HF}} & =E_{\text {int }}^{\mathrm{HF}}-E_{\text {elst }}^{(10)}-E_{\text {exch }}^{(10)}-E_{\text {ind,resp }}^{(20)}-E_{\text {exch-ind,resp }}^{(20)}
\end{aligned}
$$

The $E_{\text {elst }}^{(10)}$ is the classical (Coulombic) electrostatic energy, $E_{\text {exch }}^{(10)}$ is the exchange term that results from the antisymmetrization (symmetry adaptation) of the wave-function, $E_{\text {ind,resp }}^{(20)}$ denotes the induction (with response) energy, $E_{\text {exch-ind,resp }}^{(20)}$ is the second-order exchange-induction (with response) energy term, $E_{\mathrm{elst}}^{(10)}$ is the dispersion energy, and

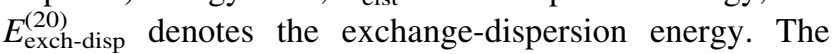
subscripts "resp" appearing in some terms indicate that this contribution was computed with orbital relaxation effects. The last term $\delta E_{\mathrm{elst}}^{(10)}$ collects the contributions to supermolecular Hartree-Fock energy beyond the second-order of intermolecular operator. The ${ }^{t} E_{\text {ind }}^{(22)}$ is the part of $E_{\text {elst }}^{(10)}$ not included in $E_{\text {ind,resp. }}^{(20)}$

The so-called full SAPT is defined by $E_{\text {int }}^{\text {SAPT2 }}$ plus some higher corrections according to the eqs:

$$
\begin{aligned}
E_{\mathrm{int}}^{\mathrm{SAPT}}= & E_{\mathrm{int}}^{\mathrm{SAPT} 2}+E_{\text {elst }, \text { resp }}^{(13)}+\left(\epsilon_{\mathrm{exch}}^{(1)}(C C S D)-\epsilon_{\mathrm{exch}}^{(1)}(2)\right) \\
& +\epsilon_{\text {disp }}^{(2)}(2)
\end{aligned}
$$

where:

$$
\begin{aligned}
\epsilon^{(n)}(k) & =\sum_{j=1}^{k} E^{(n j)} \\
\epsilon_{\mathrm{exch}}^{(1)}(\mathrm{CCSD}) & =E_{\mathrm{exch}}^{(1)}(\mathrm{CCSD})-E_{\mathrm{exch}}^{(10)}
\end{aligned}
$$

The term $\mathrm{E}_{\text {exch }}^{(1)}(\mathrm{CCSD})$ was computed with the monomer wave functions correlated at the coupled-cluster level with single and double excitation. The calculations were carried out for five complexes presented in Fig. 1 as well as for the series of complexes with different angles between the linear monomers. The interaction energy components were calculated by means of the SAPT method implemented in [12]. We used the aug-cc-pVQZ basis set in this calculations $[13,14]$.

\section{Atoms in Molecules}

The electronic density analysis, based on the Bader's Atoms in Molecules approach [15], was enabled using keywords in the Gaussian09 program package at the level of MP2/aug-cc-pVTZ, and then treated in AIM2000 program [16]. The bond critical points (BCP) were found and the electron densities and their Laplacians were calculated.

\section{Calculation of NMR properties}

Since the MBPT approach for NMR chemical shift calculations is expensive, there is a growing interest in alternatives to shielding constants calculations. DFT provides such an alternative by inclusion of correlation effects in an approximate manner with modest computational costs. This is now accepted method for studying large molecules with non-negligible correlation effects, even though this method has its own shortcomings [17].

The shielding (SC) and spin-spin coupling constants (SSCC) for all atoms were calculated on the DFT(B3LYP) level. The aug-pcS-0 basis set was used [18], as it is reported that it gives accurate results using DFT calculations. London orbitals (GIAO) [19, 20] were used to ensure the gauge-origin independence of shielding constants. The shielding constants were presented as isotropic $\left(\sigma_{\text {iso }}\right)$ and anisotropic $\left(\sigma_{\text {ani }}\right)$ part. The calculations were carried out with Gaussian03 software package.

\section{Results and discussion}

The equilibrium structures and the interaction energy

\section{Supermolecular results}

The geometry optimization for two component complexes of $\mathrm{HLi} \cdot \cdots \mathrm{HBeH}, \mathrm{FLi} \cdot \cdots \mathrm{HBeH}$ leaded to two forms-linear and cyclic. All zigzag-shaped forms converged to the 
Table 1 The interaction energies $(\mathrm{kcal} / \mathrm{mol})$ calculated by supermolecular method on the MP2/aug-cc-pVDZ level

\begin{tabular}{lrlr}
\hline & \multicolumn{1}{c}{$D_{\mathrm{e}}$} & $\Delta \mathrm{ZPE}$ & \multicolumn{1}{c}{$D_{0}$} \\
\hline BeF2 $\cdots$ LiH cyclic-C1 & -56.27 & 1.57 & -51.75 \\
BeH2 $\cdots$ LiF cyclic-C2 & -48.67 & 1.61 & -44.52 \\
BeH2 $\cdots$ LiF linear-L1 & -7.67 & 0.23 & -6.51 \\
BeH2 $\cdots$ LiH cyclic-C3 & -41.23 & 0.90 & -40.67 \\
BeH2 $\cdots$ LiH linear-L2 & -7.78 & 0.75 & -6.07 \\
\hline
\end{tabular}

cyclic form in optimization procedure. The monomers and dimers with geometry details are shown in Fig. 1. Some of the linear and cyclic complexes were previously calculated by Rozas et al. [5].

The supermolecular calculations are presented in Table 1. This table contains the corresponding binding energies $D_{\mathrm{e}}$, (i.e., the interaction energies corrected for BSSE), the harmonic zero-point vibrational energies $\triangle \mathrm{ZPV}$, and the dissociation energies $D_{0}$, which include both: BSSE and the zero-point corrections. They are in agreement with corresponding data presented by Rozas et al. [5]. The interaction energy in the linear complexes is typical for hydrogen bond. The interaction energy of the cyclic complexes is significantly larger-it is not surprising, as the interaction in this dimer is two-centered and in $\mathrm{HBeH} \cdots \mathrm{LiH}$ complex it is even impossible to determine which hydrogen atom participating in interaction belongs to which molecule. This is in line with the values of charge transfer from electron donor $\mathrm{BeH}_{2}$ to electron acceptor $\mathrm{LiH}$ or $\mathrm{LiF}$ subunits, which is larger for the cyclic than for linear complexes. Therefore, the cyclic multiple systems cannot be treated as the conventional hydrogen bond structures. Nevertheless, it is interesting to analyze different terms of the SAPT interaction energies in both type of complexes, as it advances our understanding of hydrogen bond and very strong interaction in cyclic systems.

\section{SAPT results}

As the supermolecular method gives only the overview of the interaction between molecules, it was essential to introduce SAPT calculations to have insight into nature of interaction. This method was successfully used to investigate a classical hydrogen bonds, as well as dihydrogen bonded and stacking structures [3, 4]. Table 2 presents the decomposition of interaction energy calculated by means of SAPT/aug-cc-pVQZ. Before we take a look at the individual contributions to the intermolecular energy, we shall discuss the accuracy of the SAPT results, comparing Tables 1 and 2 . The SAPT results obtained with aug-cc-pVQZ basis set show a greater stability of the cyclic configurations than the linear ones in the same basis set, in agreement with the supermolecular results. Thus, it can be concluded that the energies are calculated by both, so different methods are quite similar.

To facilitate interpretation of the numbers, Fig. 2 shows the contributions of each component to the total energy. In
Table 2 The components of the interaction energy $(\mathrm{kcal} / \mathrm{mol})$ for complexes calculated by SAPT method (using aug-cc-pVQZ basis set)

\begin{tabular}{|c|c|c|c|c|c|}
\hline Components & $\begin{array}{l}\mathrm{BeF} 2 \cdots \mathrm{LiH} \\
\mathrm{C} 1\end{array}$ & $\begin{array}{l}\mathrm{BeH} 2 \cdots \mathrm{LiF} \\
\mathrm{C} 2\end{array}$ & $\begin{array}{l}\mathrm{BeH} 2 \cdots \mathrm{LiF} \\
\mathrm{L} 1\end{array}$ & $\begin{array}{l}\mathrm{BeH} 2 \cdots \mathrm{LiH} \\
\mathrm{C} 3\end{array}$ & $\begin{array}{l}\mathrm{BeH} 2 \cdots \mathrm{LiH} \\
\mathrm{L} 2\end{array}$ \\
\hline$E_{\mathrm{elst}}^{(10)}$ & -109.02 & -105.91 & -7.31 & -93.60 & -7.28 \\
\hline$E_{\mathrm{exch}}^{(10)}$ & 89.28 & 81.63 & 3.97 & 84.31 & 4.12 \\
\hline$E_{\text {elst,resp }}^{(12)}$ & 1.61 & 2.75 & 0.42 & 1.88 & 0.38 \\
\hline$E_{\mathrm{exch}}^{(11)}$ & -0.31 & 0.25 & -0.00 & 3.30 & 0.03 \\
\hline$E_{\mathrm{exch}}^{(12)}$ & 3.36 & 7.06 & -0.12 & -1.21 & -0.10 \\
\hline$E_{\text {elst,resp }}^{(13)}$ & 0.32 & -1.07 & 0.09 & 0.77 & 0.11 \\
\hline$E_{\text {ind,resp }}^{(20)}$ & -159.04 & -103.92 & -6.96 & -122.06 & -6.96 \\
\hline$E_{\mathrm{disp}}^{(20)}$ & -15.12 & -14.43 & -0.53 & -17.22 & -0.70 \\
\hline$E_{\mathrm{ex}-\mathrm{ind}, r}^{(20)}$ & 109.17 & 68.46 & 3.28 & 60.47 & 3.24 \\
\hline$E_{\text {exch-disp }}^{(20)}$ & 5.54 & 4.79 & 0.07 & 2.44 & 0.10 \\
\hline${ }^{t} E_{\mathrm{elst}}^{(10)}$ & -4.75 & -9.70 & 0.04 & -0.82 & 0.00 \\
\hline${ }^{t} E_{\mathrm{elst}}^{(10)}$ & 3.26 & 6.39 & -0.02 & 0.41 & -0.00 \\
\hline$\epsilon_{\mathrm{exch}}^{(1)}(C C S D)$ & 3.98 & 9.61 & -0.20 & 2.90 & -0.05 \\
\hline$\epsilon_{\mathrm{exch}}^{(1)}(2)$ & 3.05 & 7.31 & -0.12 & 2.09 & -0.07 \\
\hline$\epsilon_{\mathrm{disp}}^{(2)}(2)$ & -2.92 & -2.89 & -0.14 & -3.13 & -0.17 \\
\hline$\delta_{\mathrm{int}, r}^{\mathrm{HF}}$ & -0.91 & -10.30 & -0.34 & 10.89 & -0.34 \\
\hline$E_{\mathrm{int}}^{\mathrm{SAPT} 2}$ & -76.94 & -72.93 & -7.51 & -71.21 & -7.51 \\
\hline$E_{\mathrm{int}}^{\mathrm{SAPT}}$ & -80.53 & -76.27 & -8.15 & -75.42 & -8.05 \\
\hline
\end{tabular}



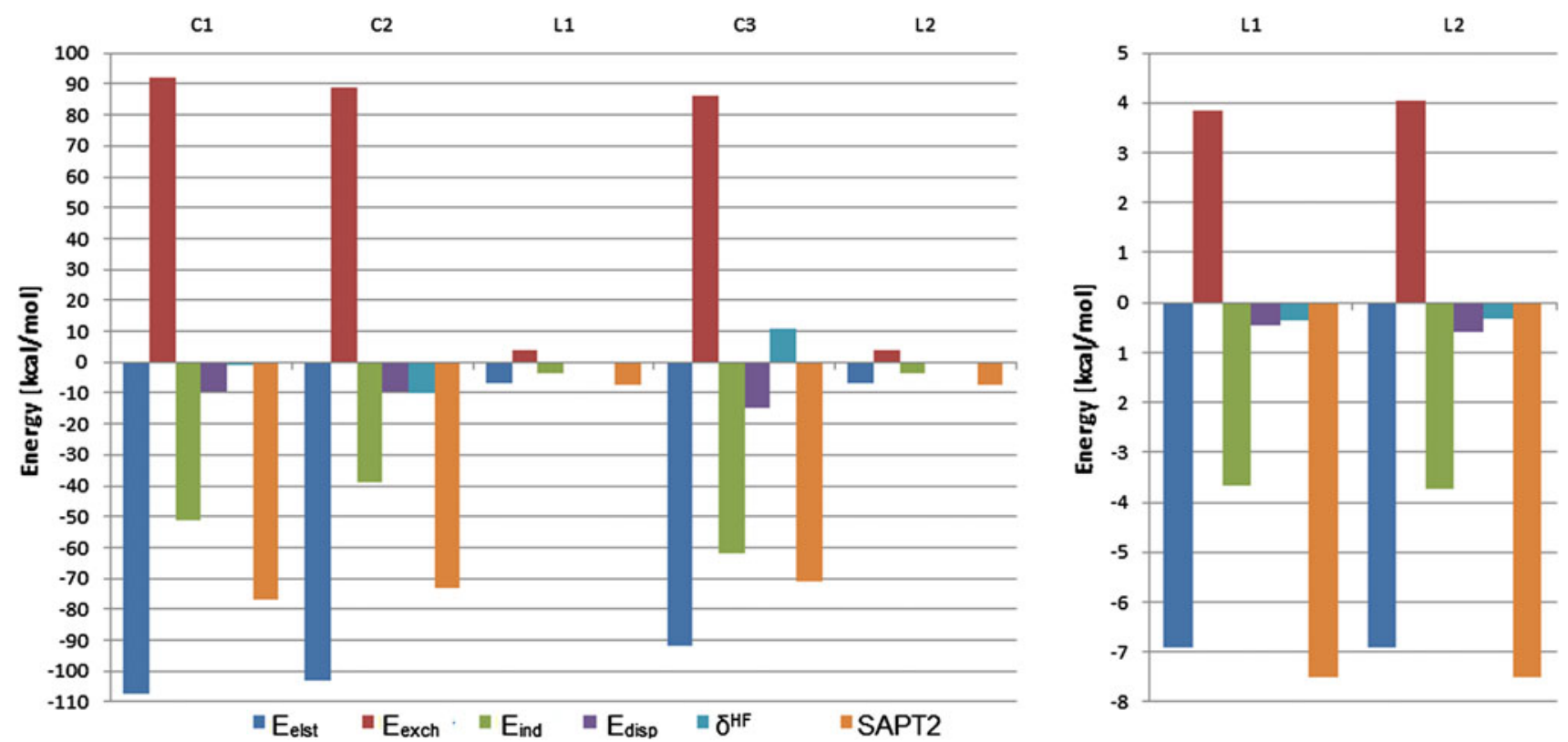

Fig. 2 Components of SAPT interaction energy (calculated using aug-cc-pVQZ basis set; right panel-repetition for L1, L2)

a search for factors which would characterize possible differences/similarities exhibited in the properties of the systems we focus on the dominant contributions. First, let us compare the decomposition for linear and cyclic complexes. The interaction-energy terms for both cyclic and linear structures differs. The dominant attraction energy originates in the electrostatic term $E_{\text {elst }}^{(10)}$. The ratio of $E_{\text {elst }}^{(10)}$ to the total SAPT energy is larger for cyclic (ca 1.3-1.4) than for the linear complexes (ca. 0.9). The first order exchange term is greater for cyclic structures than for linear ones, with the $E_{\mathrm{elst}}^{(10)} / E_{\mathrm{int}}^{\mathrm{SAPT}}$ ratio equal ca. 1.1 for cyclic, but ca. 0.5 for linear ones. On the first order, the electrostatic term overweighs the exchange effect for cyclic complexes, while this relation became reverse for linear complexes.

The second-order induction energy term $E_{\text {ind,resp }}^{(20)}$ reflects the electric polarization caused by both the charge of electron cloud and the nuclei charges. Consequently, in the traditional A-H $\cdots \mathrm{B}$ complex the larger effect the more polar the $\mathrm{X}-\mathrm{H}$ bond becomes. This term is attractive, and the ratio of this contribution to the total SAPT interaction energy is ca. 1.6-2.0 in the case of cyclic complexes, while for linear structures it is only ca. 0.8 . The $E_{\text {ind,resp }}^{(20)}$ contribution is partly compensated by repulsive $\mathrm{E}_{\mathrm{exch}-\mathrm{ind}, r}^{(20}$ term, which constitutes approximately a more than half of the $E_{\text {ind,resp }}^{(20)}$ absolute value in all complexes, but their relation to the full SAPT energies became the same.

The other attraction effect comes from dispersion $E_{\text {disp }}^{(20)}$ term. The ratio of the dispersion term to the total SAPT energy are different in two structures (ca. 0.07 for linear, and ca. 0.15 for cyclic complexes). The interplay between the induction and dispersion effects may be well characterized by analyzing the ratio of these two contributions, $E_{\text {ind }} / E_{\text {disp }}$ in the molecule-molecule interaction involving closed-shell species. The greatest ratio should indicate the system particularly favored by the induction effect. This ratio is ca. 7-10 for cyclic complexes, while it is over 13 for L1 and 9.9 for L2 complex. These data are the most noticeable difference between structures $\mathrm{C}$ and $\mathrm{L}$ (easily identified from Fig. 2.). The cyclic complexes, which have multiple interaction, are characterized by induction energy as the most important term exceeding the electrostatic term and the complexes are stable due to redistribution of electron density upon the complexation. The linear structures present different case-they have pronounced relative contribution of all energies: the electrostatic, then induction, exchange, and dispersion terms.

To find out how the interaction energy and its components change while one of monomer is moving, the additional SAPT interaction energy calculations have been carried out as a function of the angle between two monomers $\left(\mathrm{LiH}\right.$ and $\left.\mathrm{BeH}_{2}\right)$. The results are shown in Fig. 3. As the interaction is divided in components it can be observed how the nature of interaction is changing during the rotation of one monomer. It comes out that for small angles the changes are low, but for the larger ones situation dramatically changes. Starting from about $70^{\circ}$ the electrostatic, induction, and dispersion energies are significantly lowering, but the same time also the exchange repulsion is rapidly growing, what results in the positive interaction energy over $100^{\circ}$ and monomers are no longer bonded. It shows up how important and strong is the repulsion of overlapping orbitals. 


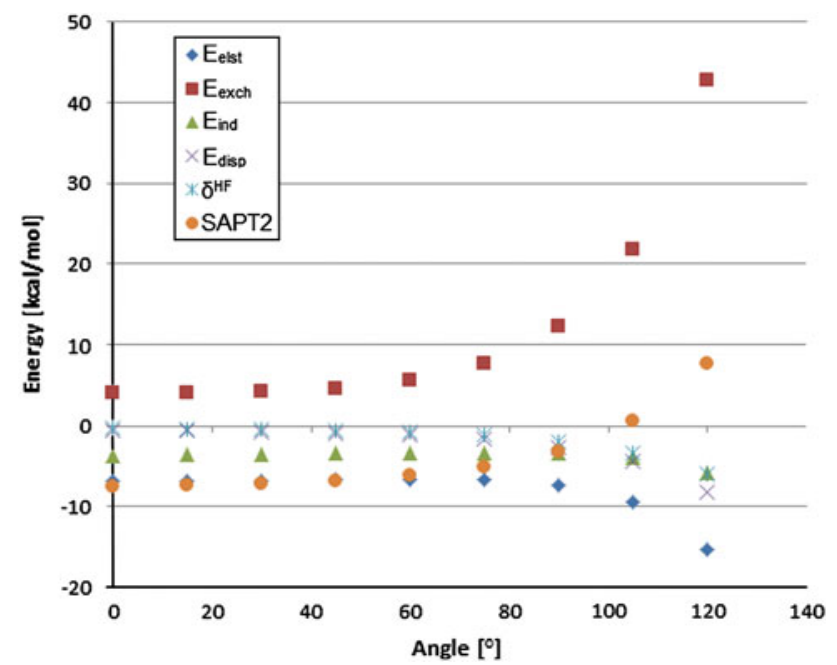

Fig. 3 SAPT components of the interaction energy as a function of angle between molecules (calculated using aug-cc-pVQZ basis set)

Finally, let us now ask the question: what is a difference between the SAPT components for the water dimer (a conventional hydrogen-bonded complex) and linear structures with hydride bonding, where hydrogen atom is a negatively charged? For the water dimer the electrostatic term exceeds the exchange energy, while dispersion term is quite close to the induction energy [21]. The SAPT decomposition of the interaction energy indicates that the ratio of the electrostatic, exchange, and induction terms to the total energy are similar in both types of hydrogen bonding, while the main difference is connected with the dispersion term. The ratio of induction to dispersion terms in conventional hydrogen bond system is ca.1.0; in contrast, studied examples of the inverse hydrogen bond are characterized by the ratio ca.10 or more. These last complexes are bound predominantly by electrostatic-exchangeinduction terms, and much less, by dispersion component.

\section{AIM analysis}

The Atoms in Molecules (AIM) analysis is important for the characterization of hydrogen bond as it was one of its criteria suggested by IUPAC [2]. AIM method [22] has became a practical tool for understanding the properties of hydrogen bonds in many cases. The topological analysis of the electron density distribution provides the evidence of a bonding interactions through the finding of a $(3,-1)$ critical point $\rho_{\mathrm{BCP}}$, which is a key topological descriptor of internuclear interactions. The Laplacian of the electron density values in bond critical point $L(A)=\int_{\omega} \mathrm{d} x$ $\left(-\frac{1}{4} \nabla^{2} \rho_{\mathrm{BCP}}\right)$ is another sensitive measure of the properties of a classical bond. Typical intermolecular hydrogen bonds can be categorized properly, as it is proofed in many papers [23], however, it should be noted that there is some

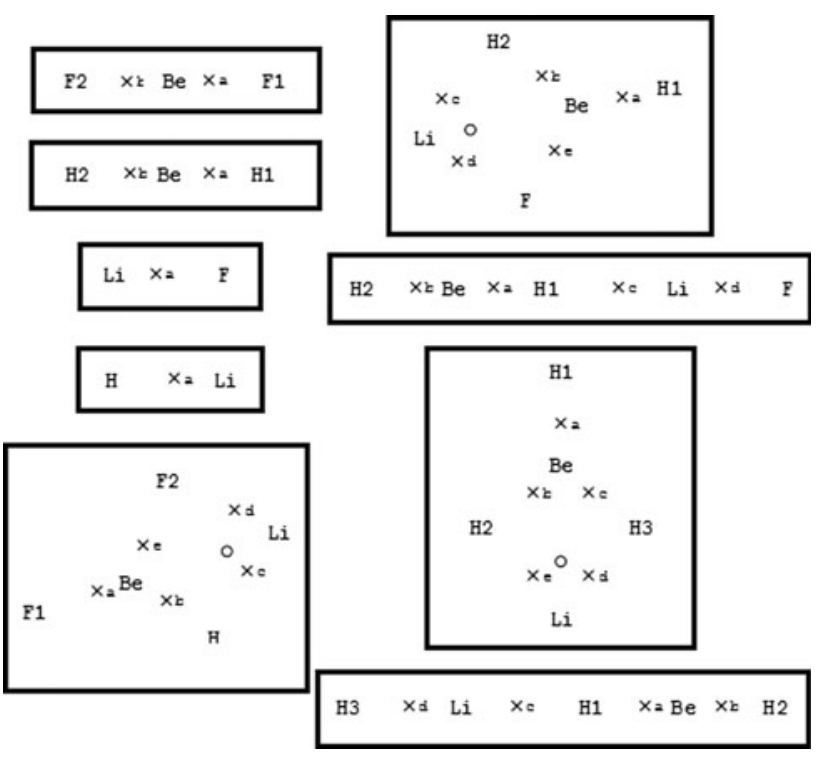

Fig. 4 The critical point positions (calculated on the MP2/augcc-pVTZ level)

controversy with regards to the use of AIM as diagnostic tool for bonding interactions [24, 25].

The Popelier criteria [22] for hydrogen bond formation, van der Waals interaction and ionic ones include: the requirement that there is the depletion of electron density charge within the atom-atom region, $\rho_{\mathrm{BCP}}$ is in the range of ca. 0.002-0.040 au.), while the value of the Laplacian at the hydrogen bond critical point $\nabla^{2} \rho_{\mathrm{BCP}}$ is positive (between 0.02 and 0.15 au.). A positive $\nabla^{2} \rho_{\text {BCP }}$ reflects an excess of kinetic energy in a bond, indicate local depletion of electron density, and this is the case in closed-shell (electrostatic) interactions. A negative Laplacian reveals excess potential energy at the BCP, what means that electronic charge in concentrated into a bond, and this is the case of covalent interactions. Fulfilling these criteria is not always mandatory [26]. For very strong hydrogen bonds like $(\mathrm{FHF})^{-}$or $\mathrm{H}_{5} \mathrm{O}_{2}^{+}$the Laplacian is negative for "intermolecular" contacts [23].

AIM calculations were preformed and their results are shown in the Fig. 4. The positions of critical point of both $(3,-1)$ and $(3,+1)$ types are shown on this figure. The numerical values of electron density $\left(\rho_{\mathrm{BCP}}\right)$ and Laplacian $\mathrm{L}(\mathrm{A})$ are presented in Table 3 . As it can be seen in the Fig. 4 one additional critical point $\mathrm{x}_{c}$ of type $(3,-1)$ appears for the linear complexes (characterized by the smallest values of $\mathrm{L}(\mathrm{A})$ ), while for cyclic complexes appears two additional $(3,-1)$ points between new contacts $\left(\mathrm{C} 1: \mathrm{x}_{b}, \mathrm{x}_{d}\right.$ or $\left.\mathrm{C} 2, \mathrm{C} 3: \mathrm{x}_{c}, \mathrm{x}_{e}\right)$. The existence of additional critical points is an evidence of forming an intermolecular bonding. The negative value of Laplacian of the electron density at the bond critical point for $\mathrm{C} 1$, $\mathrm{C} 2, \mathrm{C} 3$, as well as for $\mathrm{L} 1$ and $\mathrm{L} 2$ complexes, means the 
Table 3 Critical points characterization using AIM method for monomers and the complexes (calculated on the MP2/aug-cc-pVTZ level) ${ }^{\mathrm{a}}$

\begin{tabular}{|c|c|c|c|c|c|c|c|}
\hline Critical point type & position & $\rho_{B C P}$ & $\mathrm{~L}(\mathrm{~A})$ & Critical point type & position & $\rho_{B C P}$ & $\mathrm{~L}(\mathrm{~A})$ \\
\hline \multicolumn{4}{|l|}{$\mathrm{BeF} 2$} & \multicolumn{4}{|l|}{$\mathrm{BeH} 2$} \\
\hline$(3,-1)$ & $\mathrm{x}_{a}$ & 0.141 & -0.335 & $(3,-1)$ & $\mathrm{x}_{a}$ & 0.098 & -0.052 \\
\hline$(3,-1)$ & $\mathrm{x}_{b}$ & 0.141 & -0.335 & $(3,-1)$ & $\mathrm{x}_{b}$ & 0.098 & -0.052 \\
\hline \multicolumn{4}{|l|}{$\mathrm{LiF}$} & \multicolumn{4}{|l|}{$\mathrm{LiH}$} \\
\hline$(3,-1)$ & $\mathrm{x}_{a}$ & 0.071 & -0.174 & $(3,-1)$ & $\mathrm{x}_{a}$ & 0.036 & -0.043 \\
\hline \multicolumn{4}{|c|}{ BeF $2 \cdots$ LiH cyclic-C1 } & \multicolumn{4}{|c|}{$\mathrm{BeH} 2 \cdots \mathrm{LiF}$ cyclic-C2 } \\
\hline$(3,-1)$ & $\mathrm{x}_{a}$ & 0.127 & -0.303 & $(3,-1)$ & $\mathrm{x}_{a}$ & 0.090 & -0.057 \\
\hline$(3,-1)$ & $\mathrm{x}_{b}$ & 0.067 & -0.054 & $(3,-1)$ & $\mathrm{x}_{b}$ & 0.085 & -0.208 \\
\hline$(3,-1)$ & $\mathrm{x}_{c}$ & 0.027 & -0.033 & $(3,-1)$ & $\mathrm{x}_{c}$ & 0.042 & -0.100 \\
\hline$(3,-1)$ & $\mathrm{x}_{d}$ & 0.041 & -0.098 & $(3,-1)$ & $\mathrm{x}_{d}$ & 0.028 & -0.035 \\
\hline$(3,-1)$ & $\mathrm{x}_{e}$ & 0.085 & -0.206 & $(3,-1)$ & $\mathrm{x}_{e}$ & 0.0652 & -0.052 \\
\hline$(3,+1)$ & o & 0.023 & -0.032 & $(3,+1)$ & o & 0.023 & -0.033 \\
\hline \multicolumn{4}{|c|}{$\mathrm{BeH} 2 \cdots \mathrm{LiF}$ linear-L1 } & \multicolumn{4}{|c|}{$\mathrm{BeH} 2 \cdots \mathrm{LiH}$ linear-L2 } \\
\hline$(3,-1)$ & $\mathrm{x}_{a}$ & 0.092 & -0.058 & $(3,-1)$ & $\mathrm{x}_{a}$ & 0.088 & -0.068 \\
\hline$(3,-1)$ & $\mathrm{x}_{b}$ & 0.101 & -0.054 & $(3,-1)$ & $\mathrm{x}_{b}$ & 0.097 & -0.067 \\
\hline$(3,-1)$ & $\mathrm{x}_{c}$ & 0.013 & -0.017 & $(3,-1)$ & $\mathrm{x}_{c}$ & 0.012 & -0.018 \\
\hline$(3,-1)$ & $\mathrm{x}_{d}$ & 0.069 & -0.166 & $(3,-1)$ & $\mathrm{x}_{d}$ & 0.037 & -0.040 \\
\hline \multicolumn{8}{|c|}{$\mathrm{BeH} 2 \cdots$ LiH cyclic-C3 } \\
\hline$(3,-1)$ & $\mathrm{x}_{a}$ & 0.085 & -0.069 & & & & \\
\hline$(3,-1)$ & $\mathrm{x}_{b}$ & 0.064 & -0.062 & & & & \\
\hline$(3,-1)$ & $\mathrm{x}_{c}$ & 0.064 & -0.062 & & & & \\
\hline$(3,-1)$ & $\mathrm{x}_{d}$ & 0.027 & -0.039 & & & & \\
\hline$(3,-1)$ & $\mathrm{x}_{e}$ & 0.027 & -0.039 & & & & \\
\hline$(3,+1)$ & o & 0.024 & -0.030 & & & & \\
\hline
\end{tabular}

a The signs of $\mathrm{L}(\mathrm{A})$ in this table are opposite to the signs presented in Table 3 in Ref. [5]

evidence of the strong interactions according to criteria elaborated by AIM theory. The values of the $\left(\rho_{\mathrm{BCP}}\right)$ imply that the interaction is much stronger in the cyclic complexes, than in the linear ones. Linear structures L1 and L2 are the examples of what is called inverse hydrogen bond. These results can be rationalized by the SAPT decomposition energy data. Both linear structures are characterized by the ratio $E_{\mathrm{elst}}^{(10)} / E_{\mathrm{int}}^{\mathrm{SAPT}}$ equal ca. 0.5 , while for the cyclic structures the repulsive exchange term is more important (the ratio ca. 1.1), as well as the induction energy.

\section{Prediction of spectroscopic properties}

\section{IR spectra}

Next part of this paper is the prediction of spectroscopic properties useful for investigation on the hydrogen bond formation. As the one of the main criterion of hydrogen bond is the red-shift of the $\mathrm{X}-\mathrm{H}$ stretching frequency. However, for some $\mathrm{C}-\mathrm{H} \cdots \mathrm{Y}$ hydrogen bonds a shift to higher frequency (blue-shift) is noticed [27]. Many studies of such systems have been performed and it has been shown there are no other differences between red-shifted and blue-shifted hydrogen bonds.

We carried out the calculations of frequencies both for monomers and linear and cyclic dimers. In result of the significant geometry change, the cyclic complex frequencies show no similarity to monomer ones. The calculated frequencies of normal modes and its changes for the linear complexes are presented in Table 4.

Let us discuss now the most important frequencies for linear complexes. Our calculated difference of harmonic frequencies between the linear complexes and the monomer $\Delta v_{\mathrm{Be}-\mathrm{H}}$ is equal +67 and $+54 \mathrm{~cm}^{-1}$, for $\mathrm{HBeH} \cdots \mathrm{LiH}$ and for $\mathrm{HBeH}$. . LiF, respectively. The higher, blue-shifted value of $v_{\mathrm{Be}-\mathrm{H}}$ in the complexes, in comparison to the $\mathrm{BeH}_{2}$ monomer, means that $\mathrm{LiH}$ and $\mathrm{LiF}$ molecules stabilize the monomer unit by strengthening the $\mathrm{Be}-\mathrm{H}$ bond (one ca. $0.01 \AA$ ) and enhance the ionic nature of the $\mathrm{HBeH}$ unit. The $\mathrm{LiH}$ and $\mathrm{LiF}$ stretching modes is red-shifted by about $-9 \mathrm{~cm}^{-1}$ to lower values of frequencies in comparison with the monomer. The nature of these changes is in line with SAPT results. 
Table 4 The harmonic vibration frequencies for monomers and for linear complexes (calculated on the MP2/aug-cc-pVDZ level)

\begin{tabular}{lllr}
\hline & $v\left(\mathrm{~cm}^{-1}\right)$ & $v\left(\mathrm{~cm}^{-1}\right) \Delta v\left(\mathrm{~cm}^{-1}\right)^{\mathrm{a}}$ \\
\hline \multirow{3}{*}{$\mathrm{LiH}$} & Monomer & HBeH $\cdots \mathrm{LiH}$ & \\
& 1379 & 1370 & -9 \\
$\mathrm{BeH}_{2}$ & $735^{\mathrm{b}}$ & $704^{\mathrm{b}}$ & -31 \\
& 2050 & 2117 & 67 \\
& 2267 & 2321 & 54 \\
\hline & Monomer & $\mathrm{HBeH} \cdots \mathrm{LiF}$ & \\
$\mathrm{LiF}$ & 863 & 854 & -9 \\
& $735^{\mathrm{b}}$ & $703^{\mathrm{b}}$ & -32 \\
$\mathrm{BeH}$ & 2050 & 2117 & 67 \\
& 2267 & 2320 & 53 \\
\hline
\end{tabular}

a $v_{\text {dimer }}-v_{\text {monomer }}$

$\mathrm{b}$ Degenerated oscillation

\section{NMR results}

The main purpose of this paper is to study the changes of the NMR parameters induced by the complexation. In a relative quantity such as the binary chemical shift, the method used and the basis set effects are expected to cancel out for the most part in the complex. First, let us start the discussion on the changes of the shielding constants in linear complexes L1 and L2. They are presented in Table 5. Both linear complexes contain the intermolecular contact $\mathrm{Li} \cdots \mathrm{H} 1$, therefore the changes noticed on $\sigma(\mathrm{H} 1)$ is the most important. These shielding constants $\sigma(\mathrm{H} 1)$ increase by $4.1 \mathrm{ppm}$ (L1) or $1.9 \mathrm{ppm}$ (L2) under complexation in comparison to the monomers, in agreement with the increase the charges on these nuclei $\mathrm{H}$ participating on the intermolecular bond. It is contrary to the changes of this parameter found in many conventional hydrogen bonds. Furthermore, the formation of the cyclic complexes causes a small decrease of the $\sigma(\mathrm{H})$ shielding constant of dangling protons in $\mathrm{C} 2$ and $\mathrm{C} 3$, as well as this proton engaged in the ring.

The results of the spin-spin coupling constant calculations at B3LYP/aug-pcS-0 are illustrated in Table 6. Let us analyze first the change of the intramolecular SSCCs caused by the formation of the complexes L1 and L2. They are obtained as a difference between the SSCC in the complexes and in the monomers. In agreement with expectations, the ${ }^{1} J_{\mathrm{Be}-\mathrm{H} 1}$ in $\mathrm{L} 1$ and $\mathrm{L} 2$ complexes decreases (in term of absolute value) in respect to $\mathrm{BeH}_{2}$ under the formation of the complexes from -32.8 to -24.7 (L1) or to $-25.3 \mathrm{~Hz}$ (L2). Smaller changes are reported for this coupling in $\mathrm{C} 2$ and $\mathrm{C} 3$ structures. The hydrogen-bondtransmitted intermolecular ${ }^{1 \mathrm{H}} \mathrm{J}_{\mathrm{Li}-\mathrm{H} 1}$ coupling are positive and vary from $7.0 \mathrm{~Hz}$ (L1) to $4.9 \mathrm{~Hz}$ (L2). These values are
Table 5 NMR shielding constants (ppm) and their isotropic and anisotropy part for monomers and the complexes (calculated on the B3LYP/aug-pcS-0 level)

\begin{tabular}{|c|c|c|c|c|c|}
\hline Atom & $\sigma_{\text {iso }}(\mathrm{ppm})$ & $\sigma_{\text {ani }}(\mathrm{ppm})$ & Atom & $\sigma_{\text {iso }}(\mathrm{ppm})$ & $\sigma_{\text {ani }}(\mathrm{ppm})$ \\
\hline $\mathrm{LiH}$ & & & $\mathrm{LiF}$ & & \\
\hline $\mathrm{Li}$ & 91.6 & 14.3 & $\mathrm{Li}$ & 90.7 & 21.2 \\
\hline $\mathrm{H}$ & 26.1 & 2.4 & $\mathrm{~F}$ & 366.1 & 168.5 \\
\hline $\mathrm{BeH}_{2}$ & & & $\mathrm{BeF}_{2}$ & & \\
\hline $\mathrm{Be}$ & 91.1 & 86.3 & $\mathrm{Be}$ & 116.4 & 72.4 \\
\hline $\mathrm{H}$ & 28.3 & 3.8 & $\mathrm{~F}$ & 387.8 & 140.6 \\
\hline \multicolumn{6}{|c|}{ BeF $2 \cdots$ LiH cyclic-C1 } \\
\hline $\mathrm{Be}$ & 114.5 & 28.8 & $\mathrm{Li}$ & 91.8 & 7.4 \\
\hline F1 & 343.9 & 89.2 & $\mathrm{H}$ & 29.1 & 4.0 \\
\hline $\mathrm{F} 2$ & 326.0 & 105.2 & & & \\
\hline \multicolumn{6}{|c|}{$\mathrm{BeH} 2 \cdots \mathrm{LiF}$ cyclic-C 2} \\
\hline $\mathrm{Be}$ & 104.1 & 25.9 & $\mathrm{Li}$ & 91.6 & 7.5 \\
\hline $\mathrm{F}$ & 310.2 & 220.4 & H1 & 27.7 & 3.1 \\
\hline $\mathrm{H} 2$ & 28.4 & 1.5 & & & \\
\hline \multicolumn{6}{|c|}{$\mathrm{BeH} 2 \cdots \mathrm{LiF}$ linear-L1 } \\
\hline $\mathrm{Be}$ & 95.3 & 79.8 & $\mathrm{Li}$ & 91.1 & 23.7 \\
\hline $\mathrm{F}$ & 374.2 & 157.1 & H1 & 30.2 & 4.7 \\
\hline $\mathrm{H} 2$ & 27.8 & 4.3 & & & \\
\hline \multicolumn{6}{|c|}{$\mathrm{BeH} 2 \cdots \mathrm{LiH}$ cyclic-C3 } \\
\hline $\mathrm{Be}$ & 95.2 & 24.2 & $\mathrm{Li}$ & 87.8 & 7.6 \\
\hline H1 & 26.9 & 2.0 & $\mathrm{H} 2$ & 27.2 & 4.5 \\
\hline H3 & 27.2 & 4.5 & & & \\
\hline \multicolumn{6}{|c|}{$\mathrm{BeH} 2 \cdots \mathrm{LiH}$ linear-L2 } \\
\hline $\mathrm{Be}$ & 95.8 & 79.0 & $\mathrm{Li}$ & 89.7 & 20.2 \\
\hline H1 & 30.2 & 4.4 & $\mathrm{H} 2$ & 27.9 & 4.2 \\
\hline H3 & 26.3 & 2.9 & & & \\
\hline
\end{tabular}

similar as in the complexes with conventional hydrogen bonds.

\section{Conclusions}

The formation of the so-called inverse hydrogen bond, in which the proton atom posses excess of negative charge, changes electronic structure of the subunits. We have carried out a systematic analysis of the model complexes investigating, how these changes affect the IR spectra and the NMR parameters. The bonding in the complexes has been analyzed by Symmetry-Adapted Perturbation Theory to provide insight into the nature of the interaction. The most important results are summarized below.

1. The minima was found for linear (L1, L2) and cyclic (C1, C2, C3) structures of $\mathrm{HLi} \cdots \mathrm{HBeH}, \mathrm{FLi} \cdots \mathrm{HBeH}$ complexes. The interaction energy of the cyclic complexes is significantly larger-these systems cannot be considered as structures with hydrogen bonds. 

coupling constants for monomers and the complexes (calculated on the B3LYP/augpcS-0 level)
Table 6 NMR total spin-spin

\begin{tabular}{|c|c|c|c|c|c|c|c|}
\hline & $\mathrm{Hz}$ & & $\mathrm{Hz}$ & & $\mathrm{Hz}$ & & $\mathrm{Hz}$ \\
\hline $\mathrm{LiH}$ & & & & $\mathrm{LiF}$ & & & \\
\hline${ }^{1} J_{\mathrm{LiH}}$ & 130.2 & & & ${ }^{1} J_{\mathrm{LiF}}$ & 61.2 & & \\
\hline $\mathrm{BeH}_{2}$ & & & & $\mathrm{BeF}_{2}$ & & & \\
\hline${ }^{1} J_{\mathrm{BeH}}$ & -32.8 & ${ }^{2} J_{\mathrm{HH}}$ & 26.1 & ${ }^{1} J_{\mathrm{BeF}}$ & -28.3 & ${ }^{1} J_{F F}$ & 114.1 \\
\hline \multicolumn{8}{|c|}{$\mathrm{BeF} 2 \cdots \mathrm{LiH}$ cyclic-C1 } \\
\hline${ }^{1} J_{\mathrm{BeF} 1}$ & -19.0 & ${ }^{1} J_{\mathrm{BeF} 2}$ & 1.2 & ${ }^{1} J_{\mathrm{LiH}}$ & 23.3 & ${ }^{2} J_{\mathrm{F} 1 \mathrm{~F} 2}$ & -71.1 \\
\hline${ }^{1 \mathrm{H}} J_{\mathrm{BeH}}$ & -17.7 & ${ }^{1 \mathrm{H}} J_{\mathrm{LiF} 2}$ & 17.6 & ${ }^{2 \mathrm{H}} J_{\mathrm{BeLi}}$ & -1.2 & ${ }^{2 \mathrm{H}} J_{\mathrm{F} 2 \mathrm{H}}$ & 61.0 \\
\hline${ }^{2 \mathrm{H}} J_{\mathrm{F} 1 \mathrm{H}}$ & 19.5 & ${ }^{3 \mathrm{H}} J_{\mathrm{F} 1 \mathrm{Li}}$ & 0.2 & & & & \\
\hline \multicolumn{8}{|c|}{$\mathrm{BeH} 2 \cdots \mathrm{LiF}$ cyclic-C2 } \\
\hline${ }^{1} J_{\mathrm{BeH} 1}$ & -33.0 & ${ }^{1} J_{\mathrm{BeH} 2}$ & -14.8 & ${ }^{1} J_{\mathrm{LiF}}$ & 24.3 & ${ }^{2} J_{\mathrm{H} 1 \mathrm{H} 2}$ & 7.9 \\
\hline${ }^{1 \mathrm{H}} J_{\mathrm{H} 2 \mathrm{Li}}$ & 21.5 & ${ }^{1 \mathrm{H}} J_{\mathrm{BeF}}$ & 18.9 & ${ }^{3 \mathrm{H}} J_{\mathrm{LiH} 1}$ & -0.0 & ${ }^{2 \mathrm{H}} J_{\mathrm{BeLi}}$ & -0.9 \\
\hline${ }^{2 \mathrm{H}} J_{\mathrm{H} 2 \mathrm{~F}}$ & 34.6 & ${ }^{2 \mathrm{H}} J_{\mathrm{FH} 1}$ & 24.1 & & & & \\
\hline \multicolumn{8}{|c|}{$\mathrm{BeH} 2 \cdots \mathrm{LiF}$ linear-L1 } \\
\hline${ }^{1} J_{\mathrm{BeH} 1}$ & -24.7 & ${ }^{1} J_{\mathrm{BeH} 2}$ & -41.7 & ${ }^{1} J_{\mathrm{LiF}}$ & 63.0 & ${ }^{2} J_{\mathrm{H} 1 \mathrm{H} 2}$ & 20.1 \\
\hline${ }^{1 \mathrm{H}} J_{\mathrm{H} 1 \mathrm{Li}}$ & 7.0 & ${ }^{2 \mathrm{H}} J_{\mathrm{H} 1 \mathrm{~F}}$ & 2.3 & ${ }^{2 \mathrm{H}} J_{\mathrm{BeLi}}$ & -2.2 & ${ }^{3 \mathrm{H}} J_{\mathrm{BeF}}$ & -0.1 \\
\hline${ }^{3 \mathrm{H}} J_{\mathrm{LiH} 2}$ & 1.3 & ${ }^{4 \mathrm{H}} J_{\mathrm{FH} 2}$ & 0.3 & & & & \\
\hline \multicolumn{8}{|c|}{$\mathrm{BeH} 2 \cdots \mathrm{LiH}$ cyclic-C3 } \\
\hline${ }^{1} J_{\mathrm{BeH} 1}$ & -28.8 & ${ }^{1} J_{\mathrm{BeH} 3}$ & -12.7 & ${ }^{1} J_{\mathrm{LiH} 2}$ & 18.3 & ${ }^{2} J_{\mathrm{H} 1 \mathrm{H} 3}$ & 1.0 \\
\hline${ }^{1 \mathrm{H}} J_{\mathrm{LiH} 3}$ & 18.3 & ${ }^{1 \mathrm{H}} J_{\mathrm{BeH} 2}$ & -12.7 & ${ }^{2 \mathrm{H}} J_{\mathrm{H} 1 \mathrm{H} 3}$ & 1.0 & ${ }^{2 \mathrm{H}} J_{\mathrm{BeLi}}$ & -3.0 \\
\hline${ }^{3 \mathrm{H}} J_{\mathrm{H} 1 \mathrm{Li}}$ & 7.0 & ${ }^{2 \mathrm{H}} J_{\mathrm{H} 2 \mathrm{H} 3}$ & 4.6 & & & & \\
\hline \multicolumn{8}{|c|}{$\mathrm{BeH} 2 \cdots \mathrm{LiH}$ linear-L2 } \\
\hline${ }^{1} J_{\mathrm{BeH} 1}$ & -25.3 & ${ }^{1} J_{\mathrm{BeH} 2}$ & -41.5 & ${ }^{1} J_{\mathrm{LiH} 3}$ & 91.3 & ${ }^{2} J_{\mathrm{H} 1 \mathrm{H} 2}$ & 20.1 \\
\hline${ }^{1 \mathrm{H}} J_{\mathrm{LiH} 1}$ & 4.9 & ${ }^{2 \mathrm{H}} J_{\mathrm{BeLi}}$ & -1.0 & ${ }^{2 \mathrm{H}} J_{\mathrm{H} 1 \mathrm{H} 3}$ & 3.0 & ${ }^{3 \mathrm{H}} J_{\mathrm{LiH} 2}$ & 0.8 \\
\hline${ }^{3 \mathrm{H}} J_{\mathrm{BeH} 3}$ & -1.0 & ${ }^{4 \mathrm{H}} J_{\mathrm{H} 2 \mathrm{H} 3}$ & 0.0 & & & & \\
\hline
\end{tabular}

from $7.0 \mathrm{~Hz}$ (L1) to $4.9 \mathrm{~Hz}$ (L2), and these values are similar as in the complexes with conventional hydrogen bonds.

and L2 are bound predominantly by electrostaticexchange-induction terms, and much less, by dispersion component, while for the water dimer-a typical conventional hydrogen bond system, the electrostatic component constitutes ca $90 \%$ of the interaction energy and the induction and dispersion energies are nearly equal magnitude. These data are the most noticeable difference between the inverse and conventional hydrogen bonds.

3. The higher, blue-shifted value of $v_{\mathrm{Be}-\mathrm{H}}$ (in comparison to the $\mathrm{BeH}_{2}$ ) was found in the linear complexes. This means that $\mathrm{LiH}$ and $\mathrm{LiF}$ molecules stabilize the monomer unit by strengthening the $\mathrm{Be}-\mathrm{H}$ bond and enhance the ionic nature of the $\mathrm{HBeH}$ unit.

4. One of the main difference observed between the model inverse and conventional hydrogen bonds are the NMR shielding constants of $\sigma(\mathrm{H})$ participating in the H-bonds. The formation of the inverse hydrogen bond increase the NMR shielding values in comparison to the monomers. It is contrary to the changes of this parameter found in many conventional hydrogen bonds. Furthermore, the hydrogen-bond-transmitted intermolecular ${ }^{1 \mathrm{H}} J_{\mathrm{Li}-\mathrm{H}}$ couplings are positive and vary
Acknowledgments Computational resources were provided by the Computing Center of Faculty of Chemistry, Warsaw University. We acknowledge the computer grant G18-4 and G31-13 at ICM, Warsaw University.

Open Access This article is distributed under the terms of the Creative Commons Attribution License which permits any use, distribution, and reproduction in any medium, provided the original author(s) and the source are credited.

\section{References}

1. Hydrogen Bonding-New Insights (2006) In: Grabowski SJ, Leszczyński J (eds) Series challenges and advances in computational chemistry and physics. Springer, New York

2. Arunan E, Desiraju GR, Klein RA, Sadlej J, Scheiner S, Alkorta I, Clary DC, Crabtree RH, Dannenberg J, Hobza P, Kjaergaard HG, Legon AC, Mennucci B, Nesbitt DJ (2011) Pure Appl Chem 83:1637

3. Bakhmutov VI (2008) Dihydrogen bonds, principles, experiments, applications. Wiley, New York

4. Pecul M, Cybulski H, Sadlej J (2003) J Chem Phys 119:5094

5. Rozas I, Alkorta I, Elguero J (1997) J Phys Chem A 101:4236 
6. Alkorta I, Rozas I, Elguero J (1998) Chem Soc Rev 27:163

7. Jeziorski B, Moszyński R, Szalewicz K (1994) Chem Rev 94:1887

8. Boys SF, Bernardi F (1970) Mol Phys 19:553

9. Gaussian 03, Revision B.04, Frisch MJ, Trucks GW, Schlegel HB, Scuseria GE, Robb MA ,Cheeseman JR ,Montgomery JA Jr,Vreven T, Kudin KN , Burant JC, Millam JM, Iyengar SS , Tomasi J , Barone V, Mennucci B, Cossi M , Scalmani G, Rega N, Petersson GA, Nakatsuji H, Hada M, Ehara M, Toyota K, Fukuda R, Hasegawa J, Ishida M, Nakajima T, Honda Y, Kitao O, Nakai H, Klene M, Li X, Knox JE, Hratchian HP, Cross JB, Adamo C, Jaramillo J, Gomperts R, Stratmann RE, Yazyev O, Austin AJ, Cammi R, Pomelli C, Ochterski JW, Ayala PY, Morokuma K, Voth GA, Salvador P, Dannenberg JJ, Zakrzewski VG, Dapprich S, Daniels AD, Strain MC, Farkas O, Malick DK, Rabuck AD, Raghavachari K, Foresman JB, Ortiz JV, Cui Q., Baboul AG, Clifford S, Cioslowski J, Stefanov BB, Liu G, Liashenko A, Piskorz P, Komaromi I, Martin RL, Fox DJ, Keith T, Al-Laham MA, Peng CY, Nanayakkara A, Challacombe M, Gill PMW, Johnson B, Chen W, Wong MW, Gonzalez C, Pople JA (2003) Gaussian, Inc., Pittsburgh

10. Jeziorski B, Moszyński R, Ratkiewicz A, Rybak S, Szalewicz K, Williams HL (1993) Methods and techniques in computational chemistry: METECC-94 Ed. Clementi E STEF Cagliari

11. Jeziorska M, Jeziorski B, Cizek J (1987) Int J Quantum Chem 32:149

12. Bukowski R, Cenek W, Jankowski P, Jeziorski B, Jeziorska M, Kucharski SA, Misquitta AJ, Moszyński R, Patkowski K, Rybak
S, Szalewicz K, Williams HL, Wormer PES (2003) SAPT2002: an ab initio program for many-body symmetry-adapted perturbation theory calculations of intermolecular interaction energies. Sequential and parallel versions. University of Delaware and University of Warsaw

13. Dunning TH (1989) J Chem Phys 90:1007

14. Kendall RA, Dunning TH, Harrison RJ (1992) J Chem Phys 96:6796

15. Bader RFW (1990) Atoms in molecules. Oxford University Press, New York

16. AIM2000, University of applied sciences. Bielefeld, Germany

17. Helgaker T, Jaszunski M, Pecul M (2008) Prog Nucl Magn Reson Spectrose 53:249

18. Jensen F (2008) J Chem Theory Comput 4:719

19. Hameka HF (1962) Rev Mod Phys 34:87

20. Wolinski KJ, Hinton F, Pulay P (1990) J Am Chem Soc 112:8251

21. Szalewicz K, Bukowski R, Jeziorski B (2005) In: Dykstra C et al (eds) Theory and application of computational chemistry: the first forty years, chap 33. Elsevier, Amsterdam, p 958

22. Popelier PLA (2000) Atoms in molecules. An Introduction, vol 659. Prentice-Hall, Harlow

23. Grabowski SJ (2011) Chem Rev 111:2597

24. Poater J, Sola M, Bickelhaupt FM (2006) Chem Eur J 12:2889

25. Poater J, Sola M, Bickelhaupt FM (2006) Chem Eur J 12:2902

26. Woodford J (2007) J Phys Chem A 111:8519

27. Riley KE, Hobza P (2008) J Chem Theory Comput 4:232 